\title{
CAPILLARY MICROFLUIDICS-INTEGRATED IMPEDANCE SENSOR FOR RAPID LABEL-FREE ANTIBODY SENSING
}

\author{
F. Zang ${ }^{l}$, S. Chu ${ }^{l}$, K. Gerasopoulos ${ }^{1}, J . N$. Culver ${ }^{2}$, and R. Ghodssi $i^{1 *}$ \\ ${ }^{1}$ Department of Electrical and Computer Engineering, Institute for Systems Research, ${ }^{2}$ Institute for Bioscience and \\ Biotechnology Research and Department of Plant Science and Landscape Architecture \\ University of Maryland, College Park, USA
}

\begin{abstract}
We report on a microsystem integrating capillary microfluidics and impedance sensors for controlled on-chip nanoreceptor assembly and label-free antibody sensing. Leveraging engineered surface tension in open-channel capillary microfluidic pumps and stop valves, genetically engineered Tobacco mosaic virus-like particle (VLP) nanoreceptors are autonomously delivered and assembled onto the impedance microsensor. The evaporation-assisted VLP assembly shortens the time required for sensor functionalization to 6 minutes, a significant reduction in time compared with the VLP self-assembly process. Such VLP-functionalized impedance sensors can perform label-free antibody detection down to $10 \mathrm{ng} / \mathrm{mL}$ within 5 minutes. This platform has demonstrated great potential in on-demand portable biosensing.
\end{abstract}

\section{INTRODUCTION}

In recent years, there are growing needs for decentralized and personalized biomedical devices to better understand the body's functions in real-time for both care-takers and patients. These new incentives have promoted the development of a variety of portable electronics. Lab-on-a-chip biosensors are the most successful examples of these portable biomedical devices. They combine sensitive and selective transducers with microfluidics to facilitate complex sample delivery, manipulation and analysis on a portable platform $[1,2]$. However, most lab-on-a-chip devices rely heavily on external pumps and valves in sample analysis, which increases complexity of the final device operation. On the transduction aspect, the limited active area of a portable sensor platform often confines the amount of biorecognition elements available on sensors, compromising the sensitivity of a transducer. Therefore, it is necessary to develop high performance biological receptors and the associated sensor functionalization processes to simplify microsensor functionalization while enhancing sensor performance.

VLP, a derivative of the Tobacco mosaic virus capsid, is a high surface area biomolecule featuring a large number of genetically programmable sites on its nanorod surface [3]. Genetic modifications include cysteines to promote self-assembly onto various materials and binding peptides to enable selective target molecule binding. Previously, VLPs were successfully utilized as biosensing nanoreceptors for detecting antibodies in sandwiched immunoassays, which relied on labeling the bound target molecules with enzyme-linked secondary antibodies $[2,4]$. The labeling, necessitated most likely by low VLP assembly density, adds to the overall cost and time of sensing.

In this work, we integrate VLP bio-recognition elements on an impedance microsensor through an autonomous microfluidic platform to address the aforementioned challenges. High aspect ratio KMPR capillary micropumps and stop valves are created on top of the impedance sensor surface. Leveraging capillary action and surface evaporation, the utilization efficiency of VLPs in sensor functionalization is greatly improved compared with VLP self-assembly (which requires emersion of device surface in VLP suspension for 18 hours). An impedance microsensor was developed on the microsystem platform to study the accelerated VLP assembly process as well as real-time monitoring of biological binding events between the target antibody and VLP sensing probes. The VLPs assemble and form a dense functional receptor layer on the sensor, which increases the biosensing efficacy and enabled label-free antibody sensing rapidly after the sensor functionalization.

\section{METHODS}

\section{Capillary microfluidics-integrated impedance sensor}

The capillary microfluidics-integrated impedance sensor microsystem aims at achieving rapid as well as controlled sensor functionalization. The microsystem is composed of an impedance microsensors, three open-channel capillary micropump units and an array of microfluidic stop-valves. The benefits of using capillary microfluidic devices are the autonomous liquid sample delivery and manipulation using capillary action.

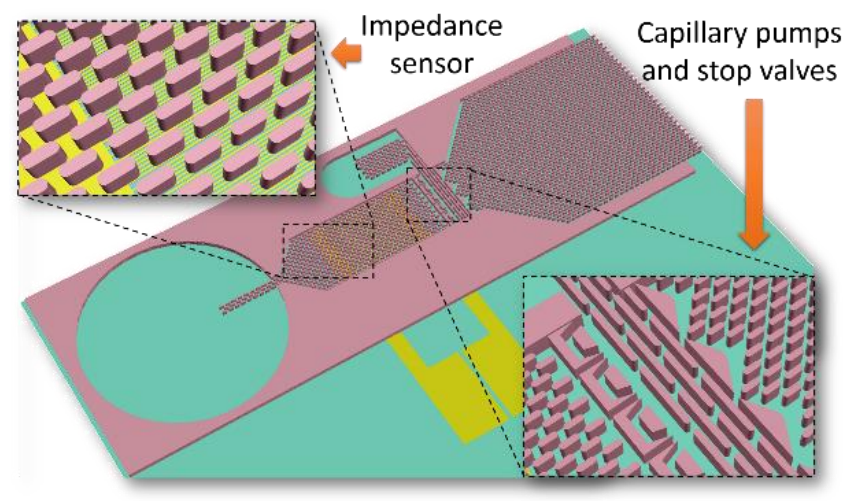

Figure 1: Schematic of the impedance sensor and capillary microfluidics-integrated microsystem.

Fig. 1 shows a schematic of our microsystem. Micropillar structures on a silicon wafer create the main geometries of the capillary micropump. The hydrophilic sidewalls of the pillar structures provide capillary driving forces to advance the liquid meniscus. The stop valve array is made of sharp "nozzles", which dramatically change the sidewall-meniscus angle during liquid filling. This creates a localized low surface energy pocket to prevent the liquid sample from flowing to the venting capillary pump on right [5]. A capillary pump that is connected to a smaller inlet provides the only triggering mechanism. For antibody sensing, a triggering fluid is introduced from the small inlet in the middle. The fluid breaks the liquid-air interface of the meniscus at the stop valves, generating continuous flow while performing antibody sensing.

The impedance microsensor features interdigitated electrodes with $4 \mu \mathrm{m}$ width/spacing and an overall area of $2 \times 2 \mathrm{~mm}^{2}$. The capillary microfluidic layer is $60 \mu \mathrm{m}$ thick with an aspect ratio (channel depth : channel width) of 2.4:1 at the micropump area and the aspect ratio of $4: 1$ at the stop valve. From theoretical calculation, the desired sidewall contact angle should be larger 
than $40^{\circ}$ in order to generate positive capillary driving force to the liquid.

\section{VLP sensing probe assembly and antibody sensing procedure}
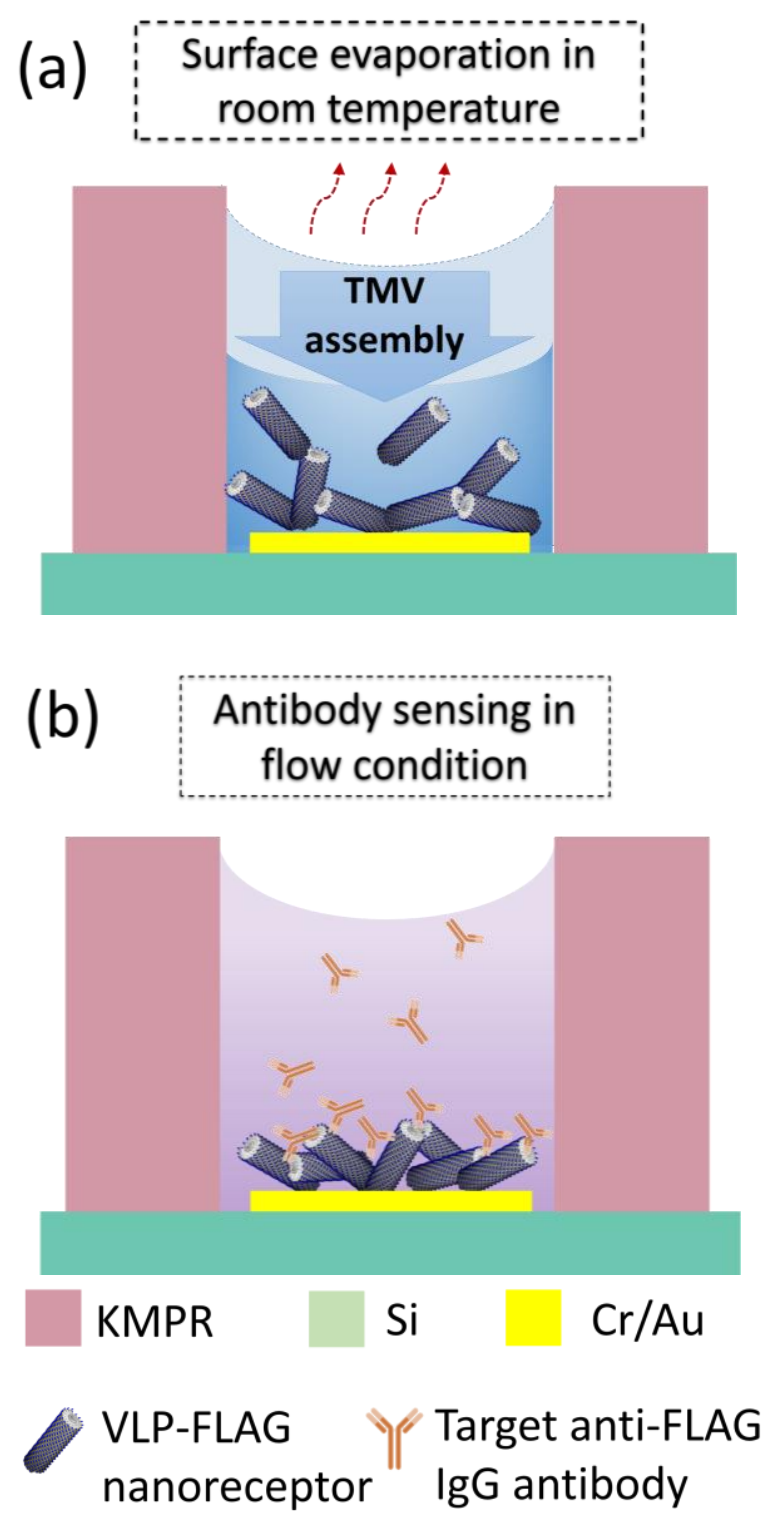

Figure 2: Schematics of (a) the evaporation-assisted VLP assembly and (b) the label-free antibody sensing process.

Fig. 2 shows the functionalization and biosensing processes, which are continuously monitored by the impedance microsensor based on the induced changes in the double layer capacitance. The VLP construct (VLP-FLAG) is genetically modified with both cysteine residues and FLAG-tag peptide sequences. In the sensor functionalization, the probes are assembled on the impedance sensor due to gold/cysteine interactions and van der Waals forces during evaporation at room temperature (Fig. 2a). Since VLP concentration increases during evaporation, an accelerated VLP assembly process and a denser VLP layer are expected.

During sensing, the binding event between the VLP sensing probes and antibody targets is directly analyzed. The triggering fluid is introduced from the small inlet in order to break the liquid- air interfacial energy at the stop-valves; thus, the antibodies can continuously flow through the functionalized impedance sensor area. The target primary anti-FLAG antibodies in $5 \mu \mathrm{L}$ of Trisbuffered saline (TBS) (1X) solution were introduced after sensor functionalization with VLPs (Fig. 2b). Antibodies show much lower dielectric constants compared to water. The substitution of water molecules by antibodies on the impedance sensor lowers the effective dielectric constants of both the double-layer and dielectric capacitances between electrodes.

\section{Microfabrication and surface treatments}

In the microfabrication of the sensor microsystem, interdigitated electrodes were fabricated using $\mathrm{Cr} / \mathrm{Au}$, the microfluidic components were made by patterning KMPR 1050 - a thick negatively photosensitive polymer layer. The challenges in the microfabrication is to simultaneously create hydrophilic sidewalls while keeping the substrate more hydrophobic. (a)

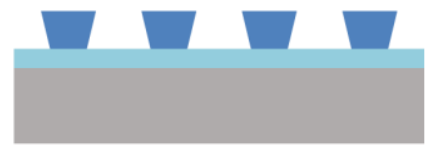

(b)

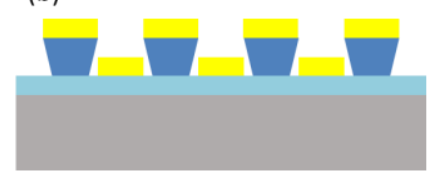

(c)

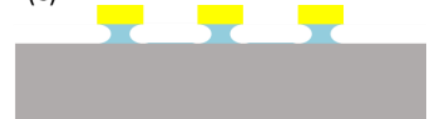

(d)

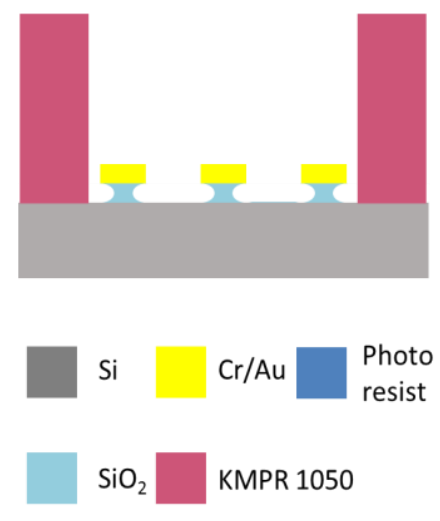

Figure 3. Microfabrication process flow of the microfluidics integrated impedimetric sensing microsystem: (a) patterning of photoresist, (b) e-beam deposition of $\mathrm{Cr} / \mathrm{Au}$, (c) lift-off of photoresist to create interdigitated impedance sensor electrodes, and etching of $\mathrm{SiO} 2$ in $\mathrm{BOE}$, and (d) creating capillary microfluidic layer through patterning of KMPR.

The fabrication process flow of the microsystem is demonstrated in Fig. 3. A $500 \mu$ m-thick silicon wafer with a 200 nm-thick $\mathrm{SiO}_{2}$ passivation layer deposited using Plasma Enhanced Chemical Vapour Deposition (PECVD) was utilized as the substrate. Negative photoresist NR9-1500PY (Futurrex Inc., NJ, USA) was spin-coated at 3000 RPM for 30 seconds to form a 1.5 $\mu \mathrm{m}$-thick photoresist layer. The photoresist was patterned using photolithography with an exposure energy dose of $280 \mathrm{~mJ} / \mathrm{cm}^{2}$ at $365 \mathrm{~nm}$ wavelength (Fig. 3a). After development, $\mathrm{Cr} / \mathrm{Au}(20$ $\mathrm{nm} / 140 \mathrm{~nm}$ ) was coated on the patterned wafer using NEXDEP Ebeam Deposition (Angstrom Engineering, Ontario, Canada) (Fig. $3 b)$. The metals deposited on the photoresist were then removed in a lift-off process in acetone, creating the impedance sensor features on the wafer. A 45 -second buffered HF etch was performed to expose the silicon (Fig. 3c) which is a more hydrophobic substrate material compared to silicon oxide.

The driving capillary force is mainly provided by the changing interfacial energy on the sidewall, which largely depends on the sidewall geometries. After impedance sensor fabrication, KMPR 1050 (MicroChem Corp., MA, USA) was spin-coated at $3000 \mathrm{rpm}$ for 30 seconds, to form a $60 \mu \mathrm{m}$-thick layer. The KMPR 
was soft-baked at $100^{\circ} \mathrm{C}$ for 20 minutes before exposure at a dose of $1000 \mathrm{~mJ} / \mathrm{cm}^{2}$ at $365 \mathrm{~nm}$ with UV light. The wafer was then postbaked at $100{ }^{\circ} \mathrm{C}$ for 4 minutes. The KMPR development process was completed in SU-8 developer for 4 minutes followed by rinsing in isopropyl alcohol. The KMPR was then patterned on the impedance sensor and formed a high aspect ratio capillary microfluidic layer (Fig. 3d).

After fabrication, the integrated sensing microsystem was exposed to $\mathrm{O}_{2}$ plasma ( $50 \mathrm{~W}, 0.5$ Torr) for 5 minutes in order to remove residue generated during the microfabrication process as well as render the KMPR microfluidic layer hydrophilic. Interestingly, owing to the exposure of silicon substrate in the BOE etching, the substrate will show more hydrophobic behaviour compared to the sidewall (with the contact angle $>60^{\circ}$ ), which agrees with observations in literature $[6,7]$. This will eventually contribute to a higher dependency of flow movement with the sidewall geometries, and a better controllability of the flow condition in the capillary microfluidic device.

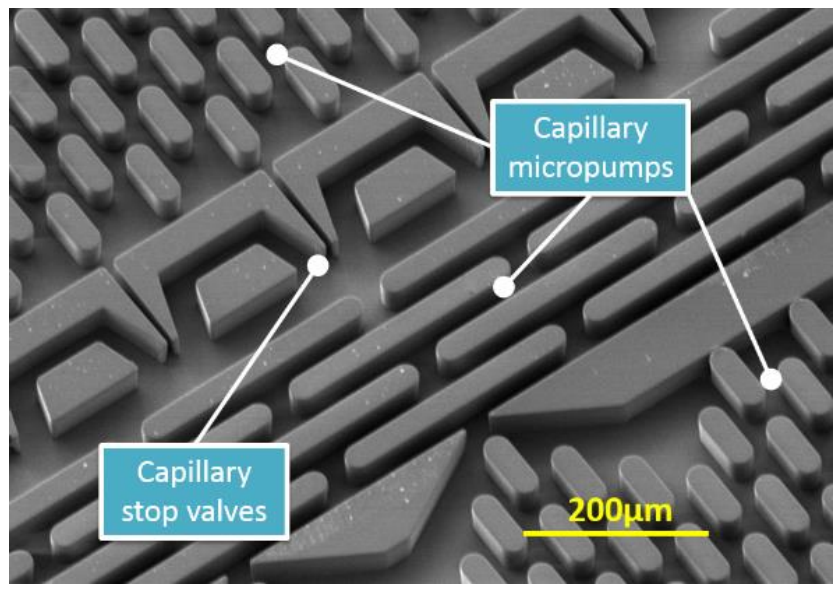

Figure 4: Scanning electron microscopy (SEM) image showing the capillary microfluidic components after microfabrication.

Fig. 4 shows the scanning electron microscopy (SEM) images of the patterned capillary micropumps and stop valves. The microfluidic components show high resolution and high aspect ratio features after one-step photolithography. The wafer was then wet etched in buffered HF (BOE) for 45 seconds to expose the silicon layer at the bottom of microfluidic channels. This can improve the controllability of the capillary microfluidic sidewall to the flow. These microfluidic components facilitate autonomous sample delivery and localized VLP assembly in the impedance sensing area. The device surface was activated using $\mathrm{O}_{2}$ plasma reactive ion etching ( $50 \mathrm{~W}, 0.5$ Torr) for 5 minutes to change the $80^{\circ}$ inherent contact angle of KMPR to $6^{\circ}$, rendering the microfluidic devices hydrophilic.

\section{RESULTS AND DISCUSSIONS}

Accelerated VLP assembly and impedance monitoring

The VLP assembly morphology was investigated using SEM, and the assembly density and coverage were studied using the ImageJ image processing tool. In order to get a good contrast in SEM imaging, the VLP-coated surfaces have gone through electroless coating of $\mathrm{Ni}$. The $\mathrm{Ni}$ coating method has been previously reported in detail in [8].

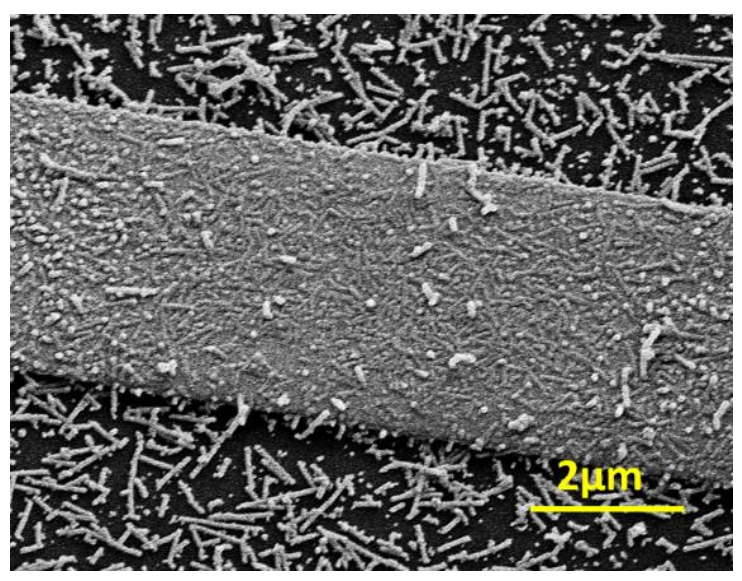

Figure 5: SEM image of the impedance sensor electrode after VLP assembly.

The SEM image in Fig. 5 validates the dense VLP coverage on the impedance sensor surface after evaporation-assisted assembly. During VLP assembly, the impedance was continuously measured. Fig. 5 also shows that the VLP-FLAG sensing probes form a dense functional layer on both the impedance sensor electrodes as well the spaces in between. The density of the evaporation enhanced VLP assembly in the open microfluidic channel is much higher compared to that previously achieved in a closed chamber [9].

The original SEM image of the VLP-FLAGs assembled on gold impedance electrodes and silicon surfaces (Fig. 5) was analyzed using ImageJ to understand the VLP surface coverage quantitatively. The original image was first processed by setting threshold values, and then converted into binary images where the white color represents VLP and the black color represents uncovered areas. The VLP coverage can then be calculated using the average grey value of each image. The specific VLP assembly on gold reaches $91 \%$ while the non-specific assembly of VLP on silicon is $41 \%$. The non-specific assembly of VLP may result from van der Waals force during evaporation-assisted assembly process. Still, there are significantly larger numbers of VLP rods assembled on the gold surface compared to the silicon surface. This validated the effect of covalent binding between the gold and thiol groups on the cysteine modified VLPs.

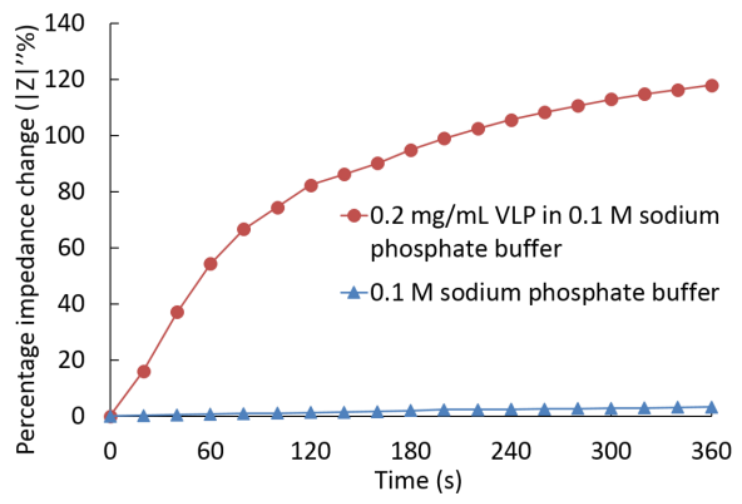

Figure 6: Percentage changes of impedance in the presence or absence of VLPS. 
Fig. 6 plots the percentage change in reactance $\left(\Delta\left|Z^{\prime \prime}\right|\right)$ during VLP assembly, showing a rapid increase to $80 \%$ within the first 2 minutes. The impedance is then gradually increased to $120 \%$ within 6 minutes, a significant reduction in time compared to the previous VLP self-assembly process [2]. In the control experiment using buffer solution, the impedance increase is less than $5 \%$, which may resulted from the fluctuation of ionic strength during evaporation of pure sodium phosphate buffer.

This impedance evolution has indicated a much more accelerated VLP assembly process through evaporation of VLPcontaining buffer solution on the impedance sensor surfaces. Previously, without evaporation, the VLP can only saturate the sensor surfaces with the same areas with a 3-hour self-assembly process [9].

\section{Rapid and label-free antibody sensing}

Specific (target) and non-specific (control) binding antibodies were introduced onto the VLP-assembled impedance sensors through the capillary microfluidic system to evaluate the biosensing efficacy.

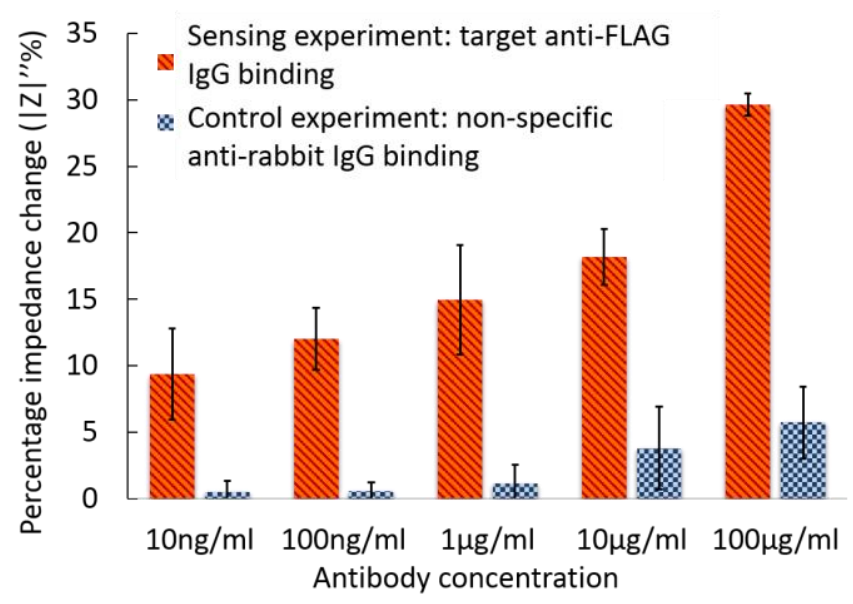

Figure 7: Percentage change in impedance for label-free sensing of target or control antibodies with increasing concentrations.

Fig. 7 shows the subsequent label-free antibody sensing using the VLP-functionalized impedance sensor. The sensor generated the maximum impedance shift over $30 \%$, and the minimum impedance increase of $9 \%$ at the lowest anti-FLAG IgG (target) concentration of $10 \mathrm{ng} / \mathrm{mL}$. In comparison, the maximum relative impedance change when introducing anti-rabbit $\operatorname{IgG}$ (control) is only $6 \%$, which is attributed to non-specific bindings between the VLP sensing probe and anti-rabbit IgG.

The VLP-based sensor can reliably detect target antibody concentrations down to $10 \mathrm{ng} / \mathrm{mL}$, equivalent to $55 \mathrm{pM}$ antibody concentrations, over a 100-fold improvement compared to the 1.2 $\mu \mathrm{g} / \mathrm{mL}$ achieved with labeling previously $[2,9]$. The sensor also showed dynamic response to a wide antibody concentration from $10 \mathrm{ng} / \mathrm{mL}$ to $100 \mu \mathrm{g} / \mathrm{mL}$. Thus, the results have demonstrated the great feasibility of the VLP-based impedance microsensors as selective, sensitivity and label-free biosensing platforms for ondemand biological analysis.

\section{CONCLUSIONS}

We developed a microsystem platform for accelerated VLP sensor functionalization and rapid biosensing. The sensor microsystem utilized capillary action to autonomously facilitated VLP assembly on an impedance sensor within 6 minutes, a significant improvement compared to the 18 -hour process in the prior art. With a densely covered VLP functional layer, the impedance microsensor was able to detect the presence of 10 $\mathrm{ng} / \mathrm{mL}$ target antibody without relying on labels.

This work is the first demonstration of using capillary microfluidic devices to control the bioreceptors assembly in order to elevate the sensitivity for label-free biosensing. The integration of impedance microsensors and capillary microfluidics provides a microsystem solution for rapid and controlled sensor functionalization. This technology offers on-demand microsensor programming with specific bioreceptors according to actual needs shortly before the sensing, keeping the optimized biosensing efficacy. Meanwhile, with the growing inventory of binding peptides, the VLPs can potentially be developed as universal vehicles for expressing high density receptor peptides in miniaturized sensor system. Therefore, the integration of VLP with microsystems will greatly benefit future applications of pointof-care diagnostics.

\section{REFERENCES}

[1] C. D. Chin, V. Linder, and S. K. Sia, "Lab-on-a-chip devices for global health: Past studies and future opportunities," Lab on a Chip, vol. 7, pp. 41-57, 2007.

[2] F. Zang, K. Gerasopoulos, K. McKinzie, J.N. Culver, and R. Ghodssi, "Autonomous Capillary Microfluidics for Rapid Nanoreceptor Assembly and Biosensing", Transducers Conference Technical Digest, Anchorage, AK, 548 (2015).

[3] A.D. Brown, L. Naves, X. Wang, R. Ghodssi, and J.N. Culver, "Carboxylate-Directed In Vivo Assembly of Viruslike Nanorods and Tubes for the Display of Functional Peptides and Residues", Biomacromolecules, 14, 3123 (2013).

[4] X.Z. Fan, L. Naves, N. P. Siwak, A.D. Brown, J.N. Culver, and R. Ghodssi, "Integration of genetically modified viruslike-particles with an optical resonator for selective biodetection", Nanotechnology, 26, 205501 (2015).

[5] M. Zimmermann, P. Hunziker, and E. Delamarche. "Valves for autonomous capillary systems." Microfluidics and Nanofluidics, 5, 395 (2008).

[6] F. Walther, P. Davydovskaya, S. Zucher, M. Kaiser, H. Herberg, A.M. Gigler and R.W. Stark, "Stability of the hydrophilic behavior of oxygen plasma activated SU-8", Journal of Micromechanics and Microengineering, 17, 524 (2007),

[7] M. Nordstrom, R. Marie, M. Calleja and A. Boisen, "Rendering SU-8 hydrophilic to facilitate use in micro channel fabrication", Journal of Micromechanics and Microengineering, 14, 1614 (2004).

[8] K. Gerasopoulos, E. Pomerantseva, M. McCarthy, A.D. Brown, C. Wang, J. N. Culver, and R. Ghodssi, "Hierarchical Three-Dimensional Microbattery Electrodes Combining Bottom-Up Self-Assembly and Top-Down Micromachining," ACS Nano, 6, 6422 (2012).

[9] F. Zang, K. Gerasopoulos, X.Z. Fan, A.D. Brown, J.N. Culver, and R. Ghodssi, "Real-Time Monitoring of Macromolecular Biosensing Probe Self-Assembly and OnChip ELISA Using Impedimetric Microsensors", Biosensors and Bioelectronics, 81, 401 (2016).

\section{CONTACT}

*R. Ghodssi, tel: +1-301-405-8158; ghodssi@umd.edu 